\title{
Mindfulness, qigong y su impacto en la salud
}

\author{
Luis A. Oblitas \\ José Anicama \\ Universidad Autónoma del Perú, \\ Lima, Perú \\ Raymundo Calderón \\ Universidad del Valle de México, \\ México \\ Robert Ferrel \\ Universidad Metropolitana, \\ Barranquilla, Colombia \\ Alexandra León \\ Universidad de la Costa, \\ Barranquilla, Colombia \\ Andrea Liliana Ortiz \\ Universidad Sergio Arboleda, \\ Santa Marta, Colombia
}

Recibido: 21 de noviembre del 2017 / Aprobado: 26 de enero del 2018

doi: 10.26439/persona2018.n021.3022

El mindfulness o atención plena tiene su origen en el budismo tibetano, y es una forma de meditación centrada en la respiración y en el escaneo corporal, que tiene un impacto significativo en el fortalecimiento de la salud y en la disminución de los síntomas de la enfermedad. El qigong es un antiguo sistema chino de origen taoísta para el mantenimiento de la salud, la curación y el aumento de la vitalidad, basado en la respiración, la postura y el movimiento. En Occidente se ha prestado más atención al mindfulness y poco interés al qigong. El propósito de este trabajo es destacar que la combinación de ambas técnicas milenarias puede brindar un mayor impacto y beneficio en la salud, ya que se correlacionan, se fundamentan en la meditación y se pueden complementar para una mayor reducción del estrés y provecho de la salud.

mindfulness / qigong / salud / estrés / enfermedad

Correo electrónico: luis.oblitas@autonoma.pe 


\section{Mindfulness, qigong and their impact on health}

Mindfulness or full attention traces its origins to Tibetan Buddhism, and constitutes a form of meditation focused on breathing and a form of body scan which significantly impacts on the strengthening of health and the decreasing of disease symptoms. Qigong is an ancient Chinese system of Taoist origin that promotes health maintenance, healing and increase in vitality, based on respiration, posture and movement. In the West, more attention has been paid to the mindfulness tradition than to the qigong. Therefore, the purpose of this article is to demonstrate that the combination of both millenary techniques may have a greater impact on and benefit to health. They both correlate, base their techniques on meditation, and their combination may prove beneficial to reduce stress and promote well-being.

\section{mindfulness / qigong / health / stress / disease}


La vida actual está llena de situaciones estresantes que afectan el bienestar, la salud y el funcionamiento de los individuos, las cuales provienen de los contextos familiares, escolares y laborales, derivadas de las demandas y exigencias de la vida moderna. Las presiones familiares, conflictos conyugales, y presiones económicas y sociales; el exceso de tareas escolares, presión social de los pares y convivencia estresante; las presiones laborales, salario insuficiente, burnout, ambiente competitivo, etcétera, definitivamente interactúan socavando la salud y el funcionamiento óptimo de los padres, hijos, alumnos, profesores y trabajadores (Grossman, Niemann, Schmidt y Walach, 2014; Good et al., 2016).

El estrés laboral es una de las variables psicosociales más investigadas. Esto se debe al impacto negativo que tiene en la salud de los trabajadores, ya que no solo afecta el rendimiento laboral, sino también a la salud integral y la calidad de vida. Más aún, cuando se convierte en estrés laboral crónico, es una fuente de síntomas y enfermedades digestivas, respiratorias, cardiacas, metabólicas e inmunes, entre otras afecciones corporales. Paralelamente, la salud psicológica del trabajador también se ve perturbada, pues se torna ansioso, depresivo, distraído, hiperactivo, irritable, voluble, falto de energía e insatisfecho con su vida laboral (Choi y Koh, 2015; Norman, Fu, Ekman, Björck y Falk, 2017).

El mindfulness (atención plena), cuyo origen se encuentra en el budismo tibetano, consiste en tener plena conciencia de sensaciones, pensamientos, sentimientos y acciones en cada momento presente. Estar en un estado consciente también puede definirse como hallarse en un estado de conciencia de sí mismo, sin juicios ni interpretaciones. Mediante esta técnica uno se acaba aceptando, mientras experimenta el momento actual, algo que se acaba consiguiendo mediante la práctica diaria de la observación pasiva de uno mismo y de sus reacciones (Wongtongkam, Krivokapic, Duncan y Bellio, 2017; Sanada et al., 2017; Schellekens et al., 2017).

El qigong es otra técnica milenaria que proviene del taoísmo. Busca fortalecer la salud, la curación y activar la energía, y se usa para el mantenimiento de la salud, la curación y el aumento de la vitalidad. Es el arte y la ciencia de la salud que utiliza técnicas de respiración, movimientos suaves y meditación para fortalecer y hacer circular el qi (energía vital). La práctica regular conduce a una mejor salud, vitalidad y una mente tranquila, sosegada y en paz (Wang, Wang, Fan, Sedas y Wang, 2017; Chan et al., 2017; Larkey et al., 2015).

$\mathrm{El}$ mindfulness y el qigong tienen varios puntos en común, y se correlacionan y complementan en términos de atención plena, conciencia y meditación, ya que cuando se realizan las actividades o ejercicios el individuo toma conciencia de las mismas en el aquí y en el ahora. Ya sea prestando atención a la respiración, el mapeo corporal, la postura y el movimiento, uno se vuelve consciente de todo lo interno y externo, y logra un mayor control y autorregulación cogntiva, emocional y conductual (Jahnke, Larkey, Rogers, Etnier y Lin, 2010; Johansson, Hassmén y Jouper, 2011; Klein, 2017). 
El objetivo de esta revisión es presentar algunas aplicaciones recientes de la meditación en la atención plena (mindfulness) y la meditación en movimiento (qigong) en poblaciones clínicas y normales, en forma específica y combinada, para el mejoramiento de la salud y el afrontamiento de la enfermedad, en las áreas clínicas, educativas y laborales. Para ello, nos apoyamos en estudios de metaanálisis y en investigaciones empíricas que aparecen en las principales publicaciones periódicas del área.

\section{Mindfulness Y SALUd}

El término inglés mindfulness proviene de la palabra sati, que a su vez procede de la lengua pali, utilizada por los budistas desde hace más de veinticinco siglos. La traducción que se le ha dado a sati es mindfulness, "atención y conciencia plena", que en las enseñanzas del Buda Gautama era uno de los factores para alcanzar el estado de iluminación (Good et at., 2016). El concepto de mindfulness está fundamentado en valores budistas, con el énfasis en cambiar los modos de pensar de mindless (sin sentido, sin conciencia) a mindful (con sentido, con conciencia) (Lomas, Medina, Ivtzan, Rupprecht y Eiroa, 2016). En psicología, el mindfulness (atención plena) consiste en observar y atender plenamente, sin distracciones ni interpretaciones, la respiración, los músculos y zonas del cuerpo (body-scan), los pensamientos, emociones y conductas (Huang, Li, Huang y Tang, 2015).

La palabra mindfulness puede usarse para describir una conducta, una práctica de cultivar la atención plena (por ejemplo, la meditación consciente), un modo o estado de conciencia, o un proceso psicológico de tipo cognitivo (Keng, Smoski y Robins, 2011). La atención plena se ha asociado con la reducción del estrés, el bienestar psicológico y la salud. Los elementos de la atención plena, es decir, la conciencia y la aceptación sin juzgar la experiencia presente del momento a momento, se consideran antídotos potencialmente eficaces contra las formas comunes del estrés (rumiación, ansiedad, preocupación, miedo, enojo, etcétera) (Bishop et al., 2004).

Mindfulness-Based Stress Reduction (MBSR) fue creado inicialmente como un programa de intervención de ocho semanas, sustentado en la enseñanza de la meditación mindfulness, respiración, escaneo corporal, yoga básico y otros métodos de relajación. El MBSR fue desarrollado por Jon Kabat-Zinn en el Centro Médico de la Universidad de Massachusetts en 1979, para enseñar a los pacientes con cáncer a reducir el estrés y el dolor. La atención plena puede definirse como "la conciencia que emerge al prestar atención, a propósito y sin prejuicios, al desarrollo de la experiencia momento por momento" (Kabat-Zinn, 2003, p. 145). Los ejercicios específicos de la atención plena pueden incluir comer consciente, caminar consciente, meditación sentada, atención en la respiración, escaneo corporal y hatha yoga (Kabat-Zinn, 1982).

Desde las primeras aplicaciones del mindfulness en la clínica, se obtuvo una reducción significativa de ansiedad y depresión (Goldin y Gross, 2010; Asmaee, Seghatoleslam, Homan, Akhvast y Habil, 2012), ansiedad y estrés (Hofmann, Sawyer, 
Witt y Oh, 2010; Sharma y Rush; 2014; Muñoz et al., 2016), dolor crónico (Hilton et al., 2017), control de estrés en diabetes (Whitebird, Kreitzer y O’Connor, 2009) y de estrés en cáncer (Sanada et al., 2017). Asimismo, contribuyó al incremento del soporte social en cáncer (Schellekens et al., 2017), la disminución de conductas de riesgo en enfermedades coronarias (Ray et al., 2014; Norman et al., 2017), disminución de la presión sanguínea (Shi et al., 2017), así como de la hiperactividad/impulsividad (Cairncross y Miller, 2016), entre otras sintomatologías.

La práctica de la atención plena ha demostrado ser útil para la mejora de la salud mental en general, ya que reduce significativamente el estrés de los empleados (Chu, 2010; Foureur, Besley, Burton, Yu y Crisp, 2013; Galantino, Baime, Maguire, Szapary y Farrar, 2005). También produce disminución de la ansiedad en el trabajo (Orzech, Shapiro, Brown y McKay, 2009; Roeser et al., 2013), reduce la depresión (Farb et al., 2010; McCraty, 2003), y provoca un mayor nivel de humor, bienestar y felicidad en empleados con un trabajo estresante (Wolever et al., 2012)

Hallazgos similares se han obtenido en profesionales de la salud (Orzech et al., 2009; McCraty, 2003). Entre ellos están la reducción del agotamiento emocional en el trabajo (Roeser et al., 2013; Hülsheger, Alberts, Feinholdt Lang, 2013), mejoramiento de las relaciones interpersonales y el rendimiento laboral (Glomb, Duffy, Bono y Yang, 2012), mayor empatía (Krasner et al., 2009), aumento de la compasión (Roeser et al., 2013), más autoeficacia (Allen, Bromley,
Kuyken y Sonnenberg, 2009), incremento del compromiso de los trabajadores (Leroy, Anseel, Dimitrova y Sels, 2013), mayor talante emocional positivo (Miner y Glomb, 2010) y disminución del estrés organizacional (Hunter y Thatcher, 2007).

La atención plena se ha asociado con el mejor desempeño laboral en los negocios de servicios (Dane y Brummel, 2013) y de los supervisores (Reb, Narayanan y Chaturvedi, 2014), en una adecuada comunicación con jefes intermedios (Shonin, Gordon, Dunn, Singh y Griffiths, 2014), incremento del rendimiento académico (Shao y Skarlicki, 2009), con conductas más éticas y prosociales (Reb, Narayanan y Ho, 2015), y la disminución de conductas antisociales (Krishnakumar y Robinson, 2015). Igualmente, se relaciona con el incremento de los comportamientos de seguridad laboral en centrales nucleares (Zhang et al., 2013), así como con trabajadores más inteligentes y experimentados (Zhang y Wu, 2014). También repercute en una atención más plena, estable y con menos errores (Smallwood y Schooler, 2015), además del control de la monotonía (Long y Christian, 2015) y de las interrupciones en el trabajo (Kuo y Yeh, 2015).

Otros estudios han señalado que la atención plena se vincula con actitudes más positivas de los líderes hacia los subordinados (Reb et al., 2014), reducción de la rumiación, autorregulación y emoción negativa (Long y Christian, 2015), mejor comunicación, reducción de conflictos, disminución de la reactividad emocional y mayor compasión y empatía (Quaglia, Goodman y Brown, 2015), así como una 
optimización en la comunicación social y emocional (Wachs y Córdova, 2007), mayor cohesión y trabajo equipo (Cleirigh y Greaney, 2014), y gestión positiva de los conflictos (Galinsky, Maddux, Gilin y White, 2008). También ayuda a la disminución del burnout laboral (Flook, Goldberg, Pinger, Bonus y Davidson, 2013), del estrés percibido (Roeser et al., 2013), de los estados de ánimo negativos (Roche, Haar y Luthans, 2014), y genera una mejora en los patrones de sueño (Hülsheger et al., 2014), mayor satisfacción y menos agotamiento en el trabajo (Hülsheger et al., 2013). Además, se ha registrado un incremento del compromiso laboral (West et al., 2014), más emociones positivas, esperanza y optimismo (Malinowski y Lim, 2015), y un desarrollo positivo en la forma de trabajar (King y Haar, 2017).

Según Good et al. (2016), la actividad de investigación de mindfulness está creciendo en forma significativa dentro de las organizaciones. Los hallazgos disponibles sugieren que la práctica de la atención plena está relacionada con varios aspectos del comportamiento organizacional en el lugar del trabajo, como la cognición, la emoción, el comportamiento y la fisiología, todo lo cual influye sobre el desempeño, las relaciones y el bienestar laboral.

Otras aplicaciones de la atención plena se han reportado en la reducción de los síntomas físicos y mejora en el bienestar psicológico de los trabajadores de un astillero (Cheong y Lee, 2014), disminución del agotamiento laboral en enfermeras (Cohen at al., 2005; Moody et al., 2013), descenso del burnout en médicos (Fortney, Luchterhand, Zakletskaia, Zgierska y Rakel, 2013; Krasner et al., 2009) y del agotamiento laboral en personal médico (Goodman y Schorling, 2012). También trae beneficios en la salud física y psicológica en médicos y enfermeras (Irving, Dobkin y Park, 2009); mejoras en el estrés percibido, calidad del sueño y la frecuencia cardiaca (Wolever $e t$ al., 2012); y mejoramiento de la atención plena y el bienestar (Niles, Vujanovic, Silberbogen, Seligowski y Potter, 2012).

En varios estudios de metaanálisis, se han comprobado mejorías en ansiedad, depresión, estilos de afrontamiento y calidad de vida (Grossman et al., 2014), disminución de la sintomatología depresiva (McCartney, Schuls y Grey, 2012), reducción del estrés, incremento de emociones positivas y mejorías en la calidad de vida (Chiesa y Serretti, 2009). También se han reportado incidencias en condiciones médicas (Greeson, 2009) y potenciación del sistema inmune (Davidson et al., 2003; Hölzel et al., 2011). En médicos y enfermeras, el mindfulness ha constribuido a la reducción del estrés, ansiedad y agotamiento (Irving et al., 2009); mejoras en el estado de ánimo y descenso del estrés laboral (Cohen et al., 2005; Mackenzie, Poulin y Seidamn, 2006). Asimismo, se advierte la otpmización de la comunicación, el manejo efectivo del estrés y el mayor control de sus vidas en las enfermeras (Frisvold, Lindquist y McAlpine, 2012); reducción del estrés y la fatiga laboral en trabajadores de la salud (Luken y Sammons, 2016); mejoras en el comportamiento organizacional (Lomas et al., 2016), y aumento del bienestar subjetivo y disminución de la reactividad emocional (Keng et al., 2011).

Otros comportamientos laborales que se han visto favorecidos con la práctica del mindfulness han sido el bienestar, rendimiento 
y compromiso laboral (Zivnuska, Kacmar, Ferguson, Dawn y Carlson, 2016). Se han encontrado mejoras en la salud ocupacional y reducción del estrés laboral (Manocha, Black, Sarris y Stough, 2011), mayor satisfacción y rendimiento laboral (Shonin et al., 2014), así como satisfacción vital, concentración en el trabajo y relaciones laborales satisfactorias (Mellor, Ingram, Van Huizen, Arnold y Harding, 2016). Igualmente, menos agotamiento y mejoría en la salud integral en enfermeras (Bazarko, Cate, Azocar y Kreitzer, 2013), disminución del burnout en médicos (Shapiro, Astin, Scott, Bishop y Córdova, 2005), atención plena y satisfacción laboral en enfermeras (Choi y Koh, 2015), disminución de la fatiga crónica y trastornos psicológicos (Huang et al., 2015), reducción del agotamiento emocional y mayor sentido de logro personal en enfermeras (Cohen et al., 2005), descenso del burnout por estrés (Mackenzie et al., 2006), y menos estrés y ansiedad en personal de oncología pediátrica (Moody et al., 2013).

En el ámbito educativo, los programas de atención plena también han resultado beneficiosos. Así, se ha logrado la reducción del estrés, el incremento de la relajación y la disminución de los trastornos del sueño en trabajadores universitarios (Wongtongkam et al., 2017); menos estrés, ansiedad y depresión en profesores de primaria (Gold et al., 2010); descenso del estrés en profesores de secundaria (Anderson, Levinson, Barker y Kiewra, 1999); menos agotamiento emocional y un mayor sentido de logro personal en maestros (Roeser et al., 2013). Asimismo, se advierte un incremento de la atención y de la resiliencia (Jennings et al., 2013), mayor atención y autocompasión en estudiantes universitarios (Newsome, Waldo y Gruszka, 2011), y mejoramiento del estado general de salud y la satisfacción vital en trabajadores administrativos de una universidad (Oblitas et al., 2017).

\section{QIGONG Y SALUD}

El qigong (chi-kung) es un método chino que combina posturas y movimientos de cuerpo, atención, concentración y respiración para aumentar la energía vital. El qigong ( $q i$ 'energía', gong 'trabajo') forma parte de la medicina tradicional china, según la cual la enfermedad surge de un desequilibrio en el flujo de la energía vital en el ser humano. Se trata de técnicas milenarias para obtener paz, salud y vitalidad, y es un método idóneo para mantenerse en forma y protegernos de la tensión y el estrés cotidiano.

Los ejercicios de qigong consiguen aumentar y mejorar la cantidad y calidad de esta energía vital y su flujo por el cuerpo; por consiguiente, establece o restablece la salud del enfermo. El qigong regula a la persona fortaleciendo su salud y previniendo enfermedades (Jahnke et al., 2010). Los ejercicios de qigong consisten en una serie de prácticas que incluyen determinadas posturas, movimiento corporal, atención plena en la respiración y la meditación, todas diseñadas para activar procesos fisiológicos y psicológicos que logren el funcionamiento vital, equilibrio, bienestar y mantenimiento o recuperación de la salud. La práctica del qigong se basa en una observación minuciosa y detallada de la naturaleza, y se fundamenta en la filosofía taoísta, el pensamiento budista y en 
la medicina china tradicional. Se la puede concebir como un arte terapéutico para mantener la salud, potenciar la vitalidad y prevenir o curar enfermedades. Se basa en los siguientes principios: (a) actitud física y psicológica relajada; (b) movimientos suaves y lentos; (c) respiración abdominal, profunda y suave; (d) atención plena en la postura, movimiento y respiración (Klein, 2017).

Yang et al. (2015) revisaron más de quinientos estudios sobre qigong y cáncer, y encontraron que el $95 \%$ de ellos informaba de un impacto favorable en esa enfermedad. Zeng, Luo, Xie, Huang y Cheng (2014) concluyeron que el qigong tuvo efectos positivos en la fatiga, el sistema inmune y los niveles de cortisol en los pacientes con cáncer.

Otras evidencias señalan que la práctica del qigong contribuye a la prevención del cáncer, ya que el ejercicio físico es un factor clave en el estilo de vida, además de potenciar el sistema inmune y mediar sobre la respuesta inflamatoria (Cheung et al., 2005; Oh et al., 2012). Disminuye la depresión y mejora la calidad del sueño (Larkey et al., 2015; Chan et al., 2017), y reduce la hipertensión arterial (Ospina et al., 2017; Lee, Lim y Lee, 2004). Mejora la calidad de vida en pacientes con cáncer (Stenlund, Lindstrom, Granlund y Burell, 2005; Pippa et al., 2007) y en pacientes con trastornos cardiovasculares (Hui, Wan, Chan y Yung, 2006; Johansson et al., 2011). Asimismo, favorece la reducción del estrés (Li y Yeh, 2005; Wang et al., 2010).

Se ha comprobado también que la práctica de qigong provoca el descenso de la ansiedad y la depresión, e incrementa el bienestar psicológico en pacientes crónicos (Rogers, Larkey y Keller, 2009; Wang et al., 2013), mejora la autoestima en los enfermos (Wang et al., 2010) y eleva el funcionamiento cognitivo (Oh et al., 2012). Asimismo, aminora el estrés en el staff de enfermeras (Griffith et al., 2008) y disminuye el dolor crónico (Holmberg, Farahaniand y Witt, 2016). Ayuda a la reducción del peso corporal en la circunferencia de la cintura, y reduce los niveles de glucosa en adultos con diabetes tipo 2 (Sun et al., 2010). Disminuye el dolor, mejora el sueño e impacta en el funcionamiento físico y psicológico en casos de fibromialgia (Sawynok y Lynch, 2014). Optimiza la función pulmonar y contribuye a la remisión de la disnea en pacientes con enfermedad pulmonar obstructiva crónica (Ding, Zhang, Li y Chen, 2014), combate la depresión y ansiedad en pacientes con el síndrome del intestino irritable, además de mejorar la calidad de vida (Wang et al., 2017).

\section{MINDFULNESS, QIGONG Y SALUD}

El qigong tiene muchos aspectos que se correlacionan con el mindfulness, ya que ambos en esencia son formas de meditación, aunque hasta el momento no se los percibe así. De esta manera, los ejercicios del qigong hacen que la persona se vuelva atenta y consciente de la interacción de los movimientos, la respiración, las posturas y los pensamientos. También se aprende a liberar y dejar fluir los pensamientos, sin juicios. En la práctica, se incorpora la mente, la respiración y el movimiento corporal, y de este modo se lleva la conciencia hacia el interior de uno mismo. Y aunque no se lo vea como una forma de meditación plena 
o consciente, la práctica de qigong tiene muchos aspectos de atención plena en sí misma (Oblitas et al., 2017).

La combinación de mindfulness $\mathrm{y}$ qigong da como resultado el incremento de energía, bienestar y alegría, la disminución del estrés y las preocupaciones, y la reducción del burnout (Jouper y Johansson, 2013). Aminora la ansiedad y la depresión, así como la presión arterial (Abbott y Lavretsky, 2013). Contribuye al mejor funcionamiento cognitivo, emocional $\mathrm{y}$ conductual (Lloyd, Tsang y Deane, 2009), al fortalecimiento del sistema inmune y la prevención de enfermedades (Burke, 2012), así como al control del dolor moderado (Zhang y Wu, 2014). Ayuda a reducir el estrés, potencia el sistema inmunológico y mejora el funcionamiento cardiovascular, respiratorio, circulatorio, linfático y digestivo (Yang et al., 2015). Mejora la salud al propiciar el ejercicio físico, la relajación y el aumento del nivel de oxígeno en sangre, además de favorecer la flexibilidad, la movilidad y la elasticidad de las articulaciones (Wang at al., 2017).

La práctica conjunta de ambas estrategias orientales tiene un efecto beneficioso sobre la mortalidad por todas las causas, la mortalidad por accidente cerebrovascular y la incidencia de accidente cerebrovascular (Wang et al., 2013). Se han probado los efectos beneficiosos del qigong sobre la presión arterial y los niveles de lípidos sanguíneos (Wang et al., 2010), así como efectos positivos en la salud, la vitalidad y una mente tranquila (Asmaee et al., 2012). Reduce el estrés en los empleados de un hospital (Bazarko et al., 2013), ralentiza el envejecimiento (Cheong y Lee, 2014), otorga mayor percepción de bienestar psicológico (Cheung et al., 2015), aumento de la densidad ósea y menos caídas en los adultos (Chan et al., 2017). Mejora la función cognitiva (Choi y Koh, 2015), aumenta el bienestar en escolares (Jennings et al., 2013), y favorece la función pulmonar en niños asmáticos (Chu, 2010).

Otras evidencias señalan que mejora la capacidad funcional cardiaca en pacientes con fibrilación auricular crónica (Abbott y Lavretsky, 2013), regula el sistema nervioso en pacientes con enfermedad coronaria (Burke, 2012), disminuye los síntomas de personas con EPOP (Ding et al., 2014), optimiza la función respiratoria (Davidson et al., 2003), reduce significativamente el nivel de glucosa en la sangre y mejora la calidad de vida de diabéticos (Cleirigh y Greaney, 2014). También disminuye la fatiga y promueve el estado de bienestar emocional de los sobrevivientes del cáncer de próstata (Crane, 2015). Reduce los efectos secundarios de la operación de cáncer de mama (Li y Yeh, 2005); disminuye los síntomas de depresión en las mujeres con cáncer de pecho durante el tratamiento de radiografía, además de tener menos fatiga y mejor calidad de vida (Hui et al., 2006); incrementa el funcionamiento del sistema inmune en los sobrevivientes del cáncer del pulmón y previene la recurrencia de los tumores (Zivnuska et al, 2016).

También se han encontrado incidencias en la reducción significativa del dolor (West et al., 2014), como el de la artrosis de rodilla (Holmberg et al., 2016) y el dolor crónico lumbar (Zeng et al., 2014); igualmente, se benefician las personas con artritis reumatoide (Chiesa y Serretti, 2009). Disminuye 
los marcadores del estrés en personas sanas (ritmo cardiaco reducido y nivel de cortisol en la saliva más bajo) (Huang et al., 2015). La práctica de qigong está relacionada con una mejora en muchos aspectos de la salud psicológica: aumenta la sensación de bienestar psicológico; reduce el estrés, ansiedad y depresión; mejora el estado de ánimo y la autoestima en los pacientes (Jouper y Johansson, 2013), y en pacientes con esclerosis múltiple se encontró mejorías significativas y consistentes respecto al equilibrio, la coordinación y los síntomas de depresión (Hilton et al., 2017).

\section{CONCLUSIONES}

El mindfulness o atención plena es un proceso psicológico inspirado en el budismo tibetano, que consiste en prestar atención total, sin juzgar, a la respiración, segmentos corporales, sensaciones, pensamientos, emociones y conductas. Se trata de alimentarse, caminar o prestar atención a cualquier estímulo estético, cultural, ecológico o citadino por el placer de observarlo y contemplarlo sin ningún tipo de elaboración cognitiva, con la finalidad de calmar y aquietar el funcionamiento cognitivo, y lograr ecuanimidad, equilibro y tranquilidad.

Dentro de un programa de intervención terapéutica de 6 a 8 sesiones de 30 a 45 minutos diarios, que combina algunas formas de atención plena en la respiración, escaneo corporal, yoga, meditación, música y descanso, se han obtenido evidencias de reducción del estrés, dolor, ansiedad, depresión; incremento del bienestar y del estado general de salud en poblaciones normales; alivio de la sintomatología de enfermedades agudas y crónicas en poblaciones clínicas; optimización en el rendimiento escolar y laboral de estudiantes y trabajadores; y mejorías en la vida social, satisfacción y calidad de vida.

El qigong o meditación en movimiento es un proceso cognitivo-conductual que involucra la atención plena en la postura corporal, respiración y movimiento de cuello, tronco y extremidades, manteniendo la concentración plena a lo largo de las rutinas para movilizar el qi o la energía vital, sin distracciones de ningún tipo. Lo común de estas técnicas orientales es la atención o meditación plena, y lo único que varía es el estímulo donde se enfoca la atención: en la respiración, en el caso del mindfulness; y en el movimiento, cuando se trata del qigong.

El qigong terapéutico, basado en la medicina china tradicional de inspiración taoísta, se ha utilizado con eficacia mayormente en estudios para reducir el dolor y el estrés en grupos de poblaciones clínicas, además de la ansiedad y la depresión en condiciones médicas, como el cáncer, trastornos cardiovasculares, diabetes y fibromialgia. En estos casos, se ha reportado una significativa disminución de la sintomatología física. En poblaciones no clínicas, se ha comprobado una importante disminución del estrés, así como mejoras en la salud y calidad de vida. Comparativamente, hay menos evidencias reportadas en trabajos científicos en Occidente; en cambio, abundan en los países asiáticos, lo que se debe al mayor interés en el mindfulness en esta parte del continente.

Consideramos que esta tendencia se igualará, ya que se han reportado varios 
estudios que ya combinan el mindfulness y el qigong para reducir sintomatología clínica en poblaciones médicas con resultados alentadores, especialmente para el control del estrés, ansiedad y depresión. Esto resulta comprensible porque es de esperar que se maximice el efecto terapéutico por la aplicación de dos técnicas basadas en la atención plena. Se podría suponer un efecto similar de esta combinación en personas sanas para potenciar y fortalecer el estado general de salud, y, por ende, el bienestar psicológico y la calidad de vida.

\section{REFERENCIAS}

Abbott, R., y Lavretsky, H. (2013). Tai Chi and Qigong for the Treatment and Prevention of Mental Disorders. The Psychiatric Clinics of North America, 36(1), 109-119. doi: 10.1016/j.psc.2013.01.011

Allen, M., Bromley, A., Kuyken, W., y Sonnenberg, S. J. (2009). Participants' Experiences of Mindfulness-Based Cognitive Therapy. Behavioural and Cognitive Psychotherapy, 37(4), 413-430. doi: 10.1017/S135246580999004X

Anderson, V. L., Levinson, E. M., Barker, W., y Kiewra, K. R. (1999). The Effects of Meditation on Teacher Perceived Occupational Stress, State and Trait Anxiety, and Burnout. School Psychology Quarterly, 14(1), 3-25. doi: 10.1037/h0088995

Asmaee, S., Seghatoleslam, T., Homan, H., Akhvast, A., y Habil, H. (2012). Effect of Mindfulness Based Stress Management on Reduction of Generalized Anxiety Disorder. Iranian Journal of Public Health, 41(10), 24-28. Recuperado de https:// www.ncbi.nlm.nih.gov/pmc/articles/PMC3494227/

Bazarko, D., Cate, R. A., Azocar, F., y Kreitzer, M. J. (2013). The Impact of an Innovative Mindfulness-Based Stress Reduction Program on the Health and Well-Being of Nurses Employed in a Corporate Setting. Journal of Workplace Behavioral Health, 28(2), 107-133. doi: 10.1080/15555240.2013.779518

Bishop, S. R., Lau, M., Shapiro, S., Carlson, L., Anderson, N. D., Carmody, J., Segal, Z. V., Abbey, S., Speca, M., Velting, D., y Devins, G. (2004). Mindfulness: A Proposed Operational Definition. Clinical Psychology, 11(3), 230-241. doi: 10.1093/clipsy.bph077

Burke, A. (2012). Comparing Individual Preferences for Four Meditation Techniques: Zen, Vipassana (Mindfulness), Qigong, and Mantra. The Journal of Science \& Healing, 8(4), 237-242. doi: 10.1016/j.explore.2012.04.003

Cheong, Y. M., y Lee, W. K. (2014). The Effects of Short-Form Mindfulness Meditation Program for Shipyard Workers on the Job Stress and Psychological Well-Being. Korean Journal Stress Research, 22(4), 181-190. doi: 10.17547/kjsr.2014.22.4.181 
Cheung, B. M., Lo, J. L., Fong, D. Y., Chan, M. Y, Wong, S. H., Wong, V. C., y Karlberg, J. P. (2005). Randomised Controlled Trial of Qigong in the Treatment of Mild Essential Hypertension. Journal of Human Hypertension, 19, 697-704. doi: 10.1038/ sj.jhh.1001884

Cairncross, M., y Miller, C. J. (2016). The Effectiveness of Mindfulness-Based Therapies for ADHD. Journal of Attention Disorders. doi: 10.1177/1087054715625301

Chan, J., Li, A., Chan, C., So, K., Chen, J., Ji, X., y Chung, L. K. (2017). Qigong Exercise Improved Quality of Sleep and Reduced Interleukin-1 Beta and Interleukin-6 among Persons with Depressive Symptoms and Sleep Disturbances. Sleep, 4(1), a405. doi: 10.1093/sleepj/zsx050.1086

Chiesa, A., y Serretti, A. (2009). Mindfulness-Based Stress Reduction for Stress Management in Healthy People. Journal of Alternative Complement Medicine, 15(5), 593-600. doi: 10.1089/acm.2008.0495.

Choi, J. I., y Koh, M. S. (2015). Relations of Job Stress, Burnout, Mindfulness and Job Satisfaction of Clinical Nurses. International Journal of Bio-Science and BioTechnology, 7(3), 121-128. doi: 10.14257/ijbsbt.2015.7.3.12

Chu, L. C. (2010). The Benefits of Meditation vis-à-vis Emotional Intelligence, Perceived Stress and Negative Mental Health. Stress and Health, 26(2), 169-180. doi: 10.1002/smi.1289

Cleirigh, D. O., y Greaney, J. (2014). Mindfulness and Group Performance. Mindfulness, 6, 601- 609. doi: 10.1007/s12671-014-0295-1

Cohen, J., Wiley, S. D., Capuano, T., Baker, D. M., Kimmel, S., y Shapiro, S. (2005). The Effects of Mindfulness-Based Stress Reduction on Nurse Stress and Burnout. Holistic Nursing Practice, 19(1), 26-35. Recuperado de https://www.ncbi.nlm.nih. gov/pubmed/15736727

Crane, R. S. (2015). Some Reflections On Being Good, On Not Being Good and On Just Being. Mindfulness, 6(5), 1226-1231. doi: 10.1007/s12671-014-0350-y

Dane, E., Bradley, J., y Brummel, B. J. (2013). Examining Workplace Mindfulness and Its Relations to Job Performance and Turnover Intention. Human Relations, 67(1), 105128. doi: $10.1177 / 0018726713487753$

Davidson, R. J., Kabat-Zinn, J., Schumacher, J., Rosenkranz, M., Muller, D., Santorelli, S., Urbanowski, F., Harrington, A., Bonus, K., y Sheridan, J. F. (2003). Alterations in Brain and Immune Function Produced by Mindfulness Meditation. Psychosomatic Medicine, 65(4), 564-570. Recuperado de https://www.ncbi.nlm.nih.gov/pubmed/12883106

Ding, M., Zhang, W., Li, K., y Chen, X. (2014). Effectiveness of T'ai Chi and Qigong on Chronic Obstructive Pulmonary Disease. Journal of Alternative and Complementary Medicine, 20(2), 79-86. doi: 10.1089/acm.2013.0087 
Duchemin, A., Steinberg, B., Marks, D. R., Vanover, K., y Klatt, M. (2015). A Small Randomized Pilot Study of a Workplace Mindfulness-Based Intervention for Surgical Intensive Care Unit Personnel. Journal of Occupational \& Environmental Medicine, 57(4), 393-399. doi: 10.1097/JOM.0000000000000371

Farb, N. S., Anderson, A. K., Mayberg, H., Bean, J., McKeon, D., y Segal, Z. V. (2010). Minding One's Emotions. Emotion, 10(1), 25-33. doi: 10.1037/a0017151

Flook, L., Goldberg, S. B., Pinger, L., Bonus, K., y Davidson, R. J. (2013). Mindfulness for Teachers. Mind, Brain and Education, 7(3). doi: 10.1111/mbe.12026

Fortney, L., Luchterhand, C., Zakletskaia, L., Zgierska, A., y Rakel, D. (2013). Abbreviated Mindfulness Intervention for Job Satisfaction, Quality of Life, and Compassion in Primary Care Clinicians. Annual Family Medicine, 11(5), 412-420. doi: 10.1370/ afm.1511

Foureur, M., Besley, K., Burton, G., Yu, N., y Crisp, J. (2013). Enhancing the Resilience of Nurses and Midwives. Contemporary Nurse, 45(1), 114-125. doi: 10.5172/ conu.2013.45.1.114.

Frisvold, M. H., Lindquist, R., y McAlpine, C. P. (2012). Living Life in the Balance at Midlife. West Journal Nurse Research, 34(2), 265-78. doi: 10.1177/0193945911424171

Galantino, M., Baime, M., Maguire, M., Szapary, P. O., y Farrar, J. T. (2005). Association of Psychological and Physiological Measures of Stress in Health-Care Professionals during an 8-Week Mindfulness Meditation Program. Journal of the International Society for the Investigation of Stress, 21, 255-261. doi: 0.1002/smi.1062

Galinsky, A. D., Maddux, W. W., Gilin, D., y White, J. B. (2008). Why It Pays to Get Inside the Head of Your Opponent. Psychological Science, 19(4), 378-384. doi: 10.1111/j.1467-9280.2008.02096.x

Glomb, T. M., Duffy, M. K., Bono, J. E., y Yang, T. (2012). Mindfulness at Work. Research in Personnel and Human Resource Management, 30, 115-157. doi: 10.1108/ S0742-7301/0000030005

Gold, E., Smith, A., Hopper, I., Herne, D., Tansey, G., y Hulland, C. (2010). MindfulnessBased Stress Reduction (MBSR) for Primary School Teachers. Journal of Child \& Family Studies, 19(2), 184-189. doi: 10.1007/s10826-009-9344-0

Goldin, P. R., y Gross, J. J. (2010). Effects of Mindfulness-Based Stress Reduction (MBSR) on Emotion Regulation in Social Anxiety Disorder. Emotion, 10(1), 83-91. doi: $10.1037 / \mathrm{a} 0018441$

Good, D. J., Lyddy, C. J., Glomb, T. M., Bono, J. E., Brown, K. W., Duffy, M. K., Baer, R. A., Brewer, J. A., y Lazar, S. W. (2016). Contemplating Mindfulness at Work. Journal of Management, 42(1), 114-142. doi: 10.1177/0149206315617003 
Goodman, M. J., y Schorling, J. B. (2012). A Mindfulness Course Decreases Burnout and Improves Well-Being among Healthcare Providers. The International Journal of Psychiatry in Medicine, 43, 119-128. doi: 10.2190/PM.43.2.b

Greeson, J. M. (2009). Mindfulness Research Update. Complementaty Health Practice Review, 14(1), 10-18. doi: 10.1177/1533210108329862

Griffith, J. M., Hasley, J. P., Liu, H., Severn, D. G., Conner, L. H., y Adler, L. E. (2008). Qigong Stress Reduction in Hospital Staff. Journal of Alternative and Complementary Medicine, 14(8), 939-945. doi: 10.1089/acm.2007.0814

Grossman, P., Niemann, L., Schmidt, S., y Walach, H. (2014). Mindfulness-Based Stress Reduction and Health Benefits. Journal of Psychosomatic Reseach, 57(1), 35-43. doi: 10.1016/S0022-3999(03)00573-7

Grover, S. L., Stephen, T. T., Teo, S. T., Pick, D., y Roche, M. (2016). Mindfulness as a Personal Resource to Reduce Work Stress in the Job Demands-Resources Model. Stress and Health, 15(5), 1-11. doi: 10.1002/smi.2726

Hilton, L., Hempel, S., Ewing, B. A., Apaydin, E., Xenakis, L., Newberry, S., Colaiaco, B., Maher, A. R., Shanman, R. M., Sorbero, M. E., y Maglione, M. A. (2017). Mindfulness Meditation for Chronic Pain. Annals of Behavioral Medicine, 51(2), 199-213. doi: $10.1007 / \mathrm{s} 12160-016-9844-2$

Hofmann, S. G., Sawyer, A. T., Witt, A. A., y Oh, D. (2010). The Effect of MindfulnessBased Therapy on Anxiety and Depression. Journal of Consulting and Clinical Psychology, 78(2), 169-183. doi: 10.1037/a0018555

Hölzel, B. K., Carmody, J., Vangel, M., Congleton, C., Yerramsetti, S. M., Gard, T., y Lazar, S. W. (2011). Mindfulness Practice Leads to Increases in Regional Brain Gray Matter Density. Psychiatry Research, 191(1), 36-43. doi: 10.1016/j.pscychresns.2010.08.006

Hui, P. N., Wan, M., Chan, W. K., y Yung, P. M. (2006). An Evaluation of Two Behavioral Rehabilitation Programs, Qigong versus Progressive Relaxation, in Improving the Quality of Life in Cardiac Patients. Journal of Alternative and Complementary Medicine, 12(4), 373-378. doi: 10.1089/acm.2006.12.373

Lomas, T., Medina, J. C., Ivtzan, I., Rupprecht, S., y Eiroa, F. J. (2017). The Impact of Mindfulness on the Well-Being and Performance of Educators. Teaching and Teacher Education, 61, 132-141. doi: 10.1016/j.tate.2016.10.008

Holmberg, C., Farahaniand, Z., y Witt, C. M. (2016). How Do Patients with Chronic Neck Pain Experience the Effects of Qigong and Exercise Therapy? Evidence-Based Complementary and Alternative Medicine. doi: 10.1155/2016/8010891

Hülsheger, U. R., Alberts, H. J., Feinholdt, A., y Lang, J. W. (2013). Benefits of Mindfulness at Work. Journal of Apllied Psychology, 98(2), 310-325. doi: 10.1037/a0031313 
Hülsheger, U. R., Lang, J. W., Depenbrock, F., Fehrmann, C., Zijlstra, F. R., y Alberts, H. J. (2014). The Power of Presence. Journal of Applied Psychology, 99(6), 1113-1128. doi: $10.1037 / \mathrm{a} 0037702$

Huang, S. L., Li, R. H., Huang, F. Y., y Tang, F. C. (2015). The Potential for MindfulnessBased Intervention in Workplace Mental Health Promotion. PLoS ONE, 10(9). doi: 10.1371/journal.pone.0138089

Hunter, L. W., y Thatcher, S. M. (2007). Feeling the Heat. Academy of Management Journal, 50, 953-968. doi: 10.5465/AMJ.2007.26279227

Irving, J. A., Dobkin, P. L., y Park, J. (2009). Cultivating Mindfulness in Health Care Professionals. Complementary Therapy Clinic Practice, 15(2), 61-66. doi: 10.1016/j. ctcp.2009.01.002

Jahnke, R., Larkey, L., Rogers, C., Etnier, J., y Lin, F. (2010). A Comprehensive Review of Health Benefits of Qigong and Tai Chi. American Journal of Health Promotion, 24(6), 1-37. doi: 10.4278/ajhp.081013-LIT-248

Jennings, P. A., Frank, J. L., Snowberg, K. E., Coccia, M. A., Mark T., y Greenberg, M. T. (2013). Improving Classroom Learning Environments by Cultivating Awareness and Resilience in Education. School Psychology Quarterly, 28(4), 374-390. doi: $10.1037 /$ spq0000035

Johansson, M., Hassmén, P., y Jouper, J. (2011). Acute Effects of Qigong Exercise on Mood and Anxiety. Sport, Exercise, and Performance Psychology, 1(S), 60-65. doi: 10.1037/2157- 3905.1.S.60

Jouper, J., y Johansson, M. (2013). Qigong and Mindfulness-Based Mood Recovery. Journal of Bodywork and Movement Therapies, 17(1), 69-76. doi: 10.1016/j.jbmt.2012.06.004

Kabat-Zinn, J. (1982). An Outpatient Program in Behavioral Medicine for Chronic Pain Patients Based on the Practice of Mindfulness Meditation. General Hospital Psychiatry, 4, 33-47. doi: 10.1016/0163-8343(82)90026-3

Kabat-Zinn, J. (2003). Mindfulness-Based Interventions in Context. Clinical Psychology, 10(2), 144-156. doi: 10.1093/clipsy.bpg016

Keng, S. L., Smoski, M. J., y Robins, C. J. (2011). Effects of Mindfulness on Psychological Health. Clinical Psychology Review, 31(6), 1041-1056. doi: 10.1016/j.cpr.2011.04.006

King, E., y Haar, J. M. (2017). Mindfulness and Job Performance: A Study of Australian Leaders. Asia Pacific Journal Human Resource, 55, 298-319. doi: 10.1111/1744-7941.12143

Klein, P. (2017). Qigong in Cancer Care. Medicines, 4(1), 2. doi: 10.3390/medicines4010002.

Krasner, M. S., Epstein, R. M., Beckman, H., et al. (2009) Association of an Educational Program in Mindful Communication with Burnout, Empathy, and Attitudes among Primary Care Physicians. JAMA, 302, 1284-1293. doi: 10.1001/jama.2009.1384 
Krishnakumar, S., y Robinson, M. D. (2015). Maintaining an Even Keel. Emotion, 15(5), 579589. doi: $10.1037 / \mathrm{emo} 0000060$

Kuo, C. Y., y Yeh, Y. Y. (2015). Reset a Task Set After Five Minutes of Mindfulness Practice. Consciousness \& Cognition, 35, 98-109. doi: 10.1016/j.concog.2015.04.023

Lee, M. S., Lim, H. J., y Lee, M. S. (2004). Impact of Qigong Exercise on Self-Efficacy and Other Cognitive Perceptual Variables in Patients with Essential Hypertension. Journal of Alternative and Complementary Medicine, 10(4), 675-680. doi: 10.1089/ acm.2004.10.675

Leroy, H., Anseel, F., Dimitrova, N. G., y Sels, L. (2013). Mindfulness, Authentic Functioning, and Work Engagement. Journal of Vocational Behavior, 82(3), 238-247. doi: 10.1016/j. jvb.2013.01.012

Larkey, L. K., Roe, D. J, Weihs, K. L., Jahnke, R., López, A. M., Rogers, C. E., Oh, B., y Guillén, J. (2015). Randomized Controlled Trial of Qigong/Tai Chi Easy on CancerRelated Fatigue in Breast Cancer Survivors. Annual Behavior Medicine, 49(2), 165-76. doi: 10.1007/s12160-014-9645-4

Li, T. Y., y Yeh, M. L. (2005). The Application of Qigong Therapy to Health Care. Hu Li Za Zhi, 52(3), 65-70. Recuperado de https://www.ncbi.nlm.nih.gov/pubmed/15986304

Long, E. C., y Christian, M. S. (2015). Mindfulness Buffers Retaliatory Responses to Injustice. Journal of Applied Psychology, 100, 1409-1422. doi: 10.1037/ap10000019

Luken, M., y Sammons, A. (2016). Systematic Review of Mindfulness Practice for Reducing Job Burnout. The American Journal of Occupational Therapy, 70(2). doi: 10.5014/ ajot.2016.016956

Lloyd, C., Tsang, H., y Deane, F. P. (2009). Qigong as a Mindful Exercise Intervention for People Living with Mental Ill Health. International Journal of Therapy and Rehabilitation, 16(7), 393-399. Recuperado de http://ro.uow.edu.au/cgi/viewcontent. cgi?article $=1242 \&$ context $=$ hbspapers

McCraty, R. M. (2003). Impact of a Workplace Stress Reduction Program on Blood Pressure and Emotional Health in Hypertensive Employees. Journal of Alternative and Complementary Medicine, 9(3), 355-369. doi: 10.1089/107555303765551589

Mackenzie, C. S., Poulin, P. A., y Seidamn, R. (2006). A Brief Mindfulness-Based Stress Reduction Intervention for Nurses and Nurse Aides. Applied Nurses Research, 19(2), 105-109. doi: 10.1016/j.apnr.2005.08.002

McCartney, R. W., Schuls, J., y Grey, A. R. (2012). Effectiveness of Mindfulness-Base Therapies in Reducing Symptoms of Depressions. European Journal of Psychotherapy \& Counselling, 14(3), 279-299. doi: 10.1080/13642537.2012.713186 
Malinowski, P., y Lim, H. J. (2015). Mindfulness at Work: Positive Affect, Hope, and Optimism Mediate the Relationship Between Dispositional Mindfulness, Work Engagement, and Well-Being. Mindfulness, 6(6), 1-13. doi: 10.1007/s12671-015-0388-5

Manocha, R., Black, D., Sarris, J., y Stough, C. (2011). A Randomized, Controlled Trial of Meditation for Work Stress, Anxiety and Depressed Mood in Full-Time Workers. Evidence-Based Complementary and Alternative Medicine, 20(11), 1-8. doi: $10.1155 / 2011 / 960583$

Mellor, J. N., Ingram, L., Van Huizen, M., Arnold, J., y Harding, A. H. (2016). Mindfulness Training and Employee Well-Being. International Journal of Workplace Health. Management, 9(2), 126-145. doi: 10.1108/IJWHM-11-2014-0049

Miner, A. G., y Glomb, T. M. (2010). State Mood, Task Performance, and Behavior at Work. Organizational Behavior and Human Decision Processes, 112, 43-57. doi: 10.1016/j. obhdp.2009.11.009

Moody, K., Kramer, D., Santizo, R. O., Magro, L., Wyshogrod, D., Ambrosio, J., Castillo, C., Lieberman, R., y Stein, J. (2013). Helping the Helpers: Mindfulness Training for Burnout in Pediatric Oncology, A Pilot Program. Journal Pediatric Oncology Nursey, 30(5), 275-84. doi: 10.1177/1043454213504497

Muñoz, R. T., Hoppes, S., Hellman, C. M., Brunk, K. L., Bragg, J. E., y Cummins, C. (2016). The Effects of Mindfulness Meditation on Hope and Stress. Research on Social Work Practice. doi: 10.1177/1049731516674319

Newsome, S., Waldo, M., y Gruszka, C. (2011). Mindfulness Group Work. The Journal for Specialidsts in Group Work, 37(4), 297-311. doi: 10.1080/01933922.2012.690832

Niles, B. L., Vujanovic, A. A., Silberbogen, K., Seligowski, A. V., y Potter, C. M. (2013). Changes in Mindfulness Following a Mindfulness Telehealth Intervention. Mindfulness, 4(4), 301-310. doi: 10.1177/1043454213504497

Norman, J., Fu, M., Ekman, I., Björck, L., y Falk, K. (2017). Effects of a Mindfulness-Based Intervention on Symptoms and Signs in Chronic Heart Failure. European Journal of Cardiovascular Nursing, 17(1), 54-65. doi: 10.1177/1474515117715843

Oblitas, L., et al. (2017). Incidencia de mindfulness y qigong sobre el estado de salud, bienestar psicológico, satisfacción vital y estrés laboral. Revista Colombiana de Psicología, 26(1), 99-113. doi: 10.15446/rcp.v26n1.54371

Oh, B., Butow, P., Mullan, B., Hale, A., Lee, M. S., Guo, X., y Clarke, S. (2012). A Critical Review of the Effects of Medical Qigong on Quality of Life, Immune Function, and Survival in Cancer Patients. Integrative Cancer Therapies, 11(2), 101-110. doi: $10.1177 / 1534735411413268$ 
Orzech, K. M., Shapiro, S. L., Brown, K., y McKay, M. (2009). Intensive Mindfulness Training-Related Changes in Cognitive and Emotional Experience. Journal of Positive Psychology, 4(3), 212-222. doi: 10.1080/17439760902819394

Ospina M. B., Bond, K., Karkhaneh, M., et al. (2007). Meditation Practices for Health. Evidence Reports/Technology Assessments, 155, 1-263. Recuperado de https://www. ncbi.nlm.nih.gov/books/NBK38360/

Pippa, L., Manzoli, L., Corti, I., Congedo, G., Romanazzi, L., y Parruti, G. (2007). Functional Capacity After Traditional Chinese Medicine (Qigong) Training in Patients with Chronic Atrial Fibrillation. Prevention Cardiology, 10(1), 22-25. doi: 10.1111/j.1520-037X.2007.05721.x

Quaglia, J. T., Goodman, R. J., y Brown, K. W. (2015). From Mindful Attention to Social Connection. Cognition and Emotion, 29(8), 1466-1474. doi: $10.1080 / 02699931.2014 .988124$

Ray, I. B., Menezes, A. R., Malur, P., Hiltbold, A. E., Reilly, J. P., y Lavie, C. J. (2014). Meditation and Coronary Heart Disease. The Ochsner Journal, 14(4), 696-703. Recuperado de https://www.ncbi.nlm.nih.gov/pmc/articles/PMC4295748/

Reb, J. M., Narayanan, J., y Chaturvedi, S. (2014). Leading Mindfully. Mindfulness, 5(1), 36-45. doi: 10.1007/s12671-012-0144-z.

Reb, J., Narayanan, J., y Ho, Z. W. (2015). Mindfulness at Work: Antecedents and Consequences of Employee Awareness and Absent-Mindedness. Mindfulness, 6, 111-122. doi: 10.1007/s12671-013-0236-4

Reid, D. (2009). Capturing Presence Moments. Canadian Journal of Occupational Therapy, 76(3), 180-188. doi: 10.1177/000841740907600307

Roche, M., Haar, J. M., y Luthans, F. (2014). The Role of Mindfulness and Psychological Capital on the Well-Being of Leaders. Journal of Occupational Health Psychology, 19(4), 476-489. doi: 10.1111/j.1467-9280.2008.02096.x

Roeser, R.W., Schonert, K. A., Jha, A., Cullen, M., Wallace, L., Wilensky, R., Oberle, E., Thomson, K., Taylor, C., y Harrison, J. (2013). Mindfulness Training and Reductions in Teacher Stress and Burnout. Journal of Educational Psychology, 105(3), 787-804. doi: $10.1037 / \mathrm{a} 0032093$

Rogers, C. E., Larkey, L. K., y Keller, C. (2009). A Review of Clinical Trials of Tai Chi and Qigong in Older Adults. West Journal Nurses Research, 31(2), 245-279. doi: $10.1177 / 0193945908327529$

Sanada, K., Alda-Díez, M., Salas Valero, M., Pérez-Yus, M. C., Demarzo, M. M. P., Montero-Marín, J., García-Toro, M., y García-Campayo, J. (2017). Effects of Mindfulness-Based Interventions on Biomarkers in Healthy and Cancer Populations. 
BMC Complementary and Alternative Medicine, 17, 125. doi: doi.org/10.1186/ s12906-017-1638-y

Sawynok, J., y Lynch, M. (2014). Qigong and Fibromyalgia. Evidence-Based Complementary and Alternative Medicine, eCAM, 379715. doi: 10.1155/2014/379715

Schellekens, M., Tamagawa, R., Labelle, L. E., Speca, M., Stephen, J., Drysdale, E., Sample, S., Pickering, B., Dirkse, D., Savage L., y Carlson, L. E. (2017). Mindfulness-Based Cancer Recovery (MBCR) versus Supportive Expressive Group Therapy (SET) for Distressed Breast Cancer Survivors. Journal Behavior Medicine, 40, 414-422. doi: 10.1007/s10865-016-9799-6

Shao, R., y Skarlicki, D. P. (2009). The Role of Mindfulness in Predicting Individual Performance. Canadian Journal of Behavioural Science, 41, 195-201. doi: 10.1037/ a0015166

Shapiro, S. L., Astin, J. A., Scott, R., Bishop, S. R., y Córdova, M. (2005). Mindfulness-Based Stress Reduction for Health Care Professionals. International Journal of Stress Management, 12(2), 164-176. doi: 10.1037/1072-5245.12.2.164

Sharma, M., y Rush, M. A. (2014). Mindfulness-Based Stress Reduction as a Stress Management Intervention for Healthy Individuals. Evidence-Based Complementary and Alternative Medicine, 19(4), 271-286. doi: 10.1177/2156587214543143

Shi, L., Zhang, D., Wang, L., Zhuang, J., Cook, R., y Chen, L. (2017). Meditation and blood pressure. Journal of Hypertension, 35(4), 696-706. doi: 10.1097/HJH.0000000000001217

Shonin, E., Gordon, W., Dunn, T, J., Singh, N. N., y Griffiths, M. D. (2014). Meditation Awareness Training (MAT) for Work-related Well-Being and Job Performance. International Journal of Mental Health \& Addiction, 12(6), 806-823. doi: 10.1007/s11469-014-9513-2

Smallwood, J., y Schooler, J. W. (2015). The Science of Mind Wandering. Annual Review of Psychology, 66, 487-518. doi: 10.1146/annurev-psych-010814-015331

Stenlund, T., Lindstrom, B., Granlund, M., y Burell, G. (2005). Cardiac Rehabilitation for the Elderly. European Journal Cardiovascular Prevention Rehabilitation, 12(1), 5-11. Recuperado de https://www.ncbi.nlm.nih.gov/pubmed/15703500

Sun, G., Lovejoy, J. C., Gillham, S., Putiri, A., Sasagawa, M., y Bradley, B. (2010). Effects of Qigong on Glucose Control in Type 2 Diabetes. Diabetes Care, 33(1), e8 e8. doi: $10.2337 / \mathrm{dc} 09-1543$

Wachs, K., y Córdova, J. V. (2007). Mindful Relating. Journal of Marital and Family Therapy, 33(4), 464-481. doi: 10.1111/j.1752-0606.2007.00032.x

Wall, R. B. (2005). Tai Chi and Mindfulness-Based Stress Reduction in a Boston Public Middle School. Journalof Pediatric Health Care, 19(4), 230-237. doi: 10.1016/j. pedhc.2005.02.006 
Wang, C., Bannuru, R., Ramel, J., Kupelnick, B., Scott, T., y Schmid, C. H. (2010). Tai Chi on Psychological Well-Being. Journal of Complementary and Alternative Medicine, 10, 23. doi: 10.1186/1472-6882-10-23

Wang, F., Man, J., Lee, E., Wu, T., Benson, H., Fricchione, G. L., y Wang, W. (2013). The Effects of Qigong on Anxiety, Depression, and Psychological Well-Being. Evidence Based Complementary and Alternative Medicine. doi: 10.1155/2013/152738

Wang, W., Wang, F., Fan, F., Sedas, A. C., y Wang, J. (2017). Mind-Body Interventions for Irritable Bowel Syndrome Patients in the Chinese Population. International Journal of Behavioral Medicine, 24(2), 191-204. doi: 10.1007/s12529-016-9589-0

West, C. P., Dyrbye, L. N., Rabatin, J. T., Call, T. G., Davidson, J. H., Multari, A., Romanski, S. A., Hellyer, J. M., Sloan, J. A., y Shanafelt, T. D. (2014). Intervention to Promote Physician Well-Being, Job Satisfaction, and Professionalism. Internal Medicine, 174(4), 527-533. doi: 10.1001/jamainternmed

Whitebird, R. R., Kreitzer, M. J., y O’Connor, P. J. (2009). Mindfulness-Based Stress Reduction and Diabetes. Diabetes Spectrum, 22(4), 226-230. doi: 10.2337/diaspect.22.4.226

Wolever, R. Q., Bobinet, K. J., McCabe, K., Mackenzie, E. R., Fekete, E., Kusnick, C. A., y Baime, M. (2012). Effective and Viable Mind-Body Stress Reduction in the Workplace. Journal Occupational Health Psychology, 17(2), 246-58. doi: 10.1037/a0027278

Wongtongkam, N., Krivokapic, B., Duncan, R., y Bellio, M. (2017). The Influence of a Mindfulness-Based Intervention on Job Satisfaction and Work-Related Stress and Anxiety. International Journal of Mental Health Promotion, 19(3), 134-143. doi: 10. 1080/14623730.2017.1316760

Yang, G. Y., Wang, L. Q., Ren, J., Zhang, Y., Li, M. L., Zhu, Y. T., Luo, J., Cheng, Y. J., Li, W. Y., Wayne, P. M., y Liu, J. P. (2015). Evidence Base of Clinical Studies on Tai Chi. PLoS ONE, 10(3), e0120655. doi: 10.1371/journal.pone.0120655

Zeng, Y., Luo, T., Xie, H., Huang, M., y Cheng, A. S. (2014). Health Benefits of Qigong or Tai Chi for Cancer Patients. Complementary Therapy Medicine, 22(1), 173-186. doi: 10.1016/j.ctim.2013.11.010

Zhang, J., Ding, W., y Wu, C. (2013). Task Complexity Natters. Personality and Individual Differences, 55(4), 433-439.

Zhang, J., y Wu, C. (2014). The Influence of Dispositional Mindfulness on Safety Behaviors. Accident Analysis \& Prevention, 70, 24-32. doi: 10.1016/j.aap.2014.03.006

Zivnuska, S., Kacmar, K. M., Ferguson, M., Dawn, S., y Carlson, D. S. (2016). Mindfulness at Work: Resource Accumulation, Well-Being, and Attitudes. Career Development International, 21(2), 106-124. doi: 10.1108/CDI-06-2015-0086 